KUNS-1966

CALT-68-2555

hep-th/0504184

\title{
Boundary and Midpoint Behaviors of Lump Solutions in Vacuum String Field Theory
}

\author{
Hiroyuki Hata * \\ Department of Physics, Kyoto University, Kyoto 606-8502, Japan \\ and \\ Sanefumi Moriyama ${ }^{\dagger}$ \\ California Institute of Technology 452-48, Pasadena, CA91125, USA \\ and \\ Graduate School of Mathematics, Nagoya University \\ Nagoya 464-8602, Japan ${ }^{\ddagger}$
}

April, 2005

\begin{abstract}
We discuss various issues concerning the behaviors near the boundary $(\sigma=0, \pi)$ and the midpoint $(\sigma=\pi / 2)$ of the open string coordinate $X(\sigma)$ and its conjugate momentum $P(\sigma)=-i \delta / \delta X(\sigma)$ acting on the matter projectors of vacuum string field theory. Our original interest is in the dynamical change of the boundary conditions of the open string coordinate from the Neumann one in the translationally invariant backgrounds to the Dirichlet one in the D-brane backgrounds. We find that the Dirichlet boundary condition is realized on a lump solution only partially and only when its parameter takes a special value. On the other hand, the string midpoint has a mysterious property: it obeys the Neumann (Dirichlet) condition in the translationally invariant (lump) background.
\end{abstract}

*hata@gauge.scphys.kyoto-u.ac.jp

${ }^{\dagger}$ moriyama@math.nagoya-u.ac.jp

$\ddagger$ Permanent address 


\section{Introduction}

One of the most celebrated catch-phrases for string field theory (SFT) is the background independence. Although perturbative string theory is constructed on a particular background, SFT is expected to connect different backgrounds by reexpanding the string field around the classical solution representing the new background. Hence, we expect that, with SFT we are able to understand how physics around the background changes dynamically.

An ideal laboratory for this interesting mechanism is the open string sector. According to Sen [1], the tachyon in a certain open string theory with D-branes indicates the instability of the D-brane. After the tachyon condensates, we arrive at states of lower dimensional D-branes and finally at the true vacuum without D-branes (tachyon vacuum). In the SFT description [2, the theories before and after the tachyon condensation, namely, the theories with D-branes of various dimensions and the one without any D-branes are connected simply by the shift of the string field $\Phi ; \Phi \rightarrow \Phi_{C}+\Phi$ with $\Phi_{C}$ being a classical solution of SFT. In this sense, the action of SFT is common among the backgrounds.

This background independence of open SFT, however, leads to an apparent contradiction as we shall explain (let us consider the bosonic open string theory in the following). The open string field $\Phi\left[X^{\mu}(\sigma), c(\sigma), b(\sigma)\right]$ is a functional of the space-time string coordinate $X^{\mu}(\sigma)$ as well as the (anti-)ghost coordinates, $c(\sigma)$ and $b(\sigma)$. Before the tachyon condensation (i.e., in open SFT in the presence of a space-filling D25-brane), the string coordinate $X^{\mu}(\sigma)$ and its conjugate momentum $P_{\mu}(\sigma)=-i \delta / \delta X^{\mu}(\sigma)$ are subject to the Neumann boundary condition (BC) for all $\mu=0,1, \cdots, 25$ :

$$
\left.\frac{d X^{\mu}(\sigma)}{d \sigma}\right|_{\sigma=0, \pi}=\left.\frac{d P_{\mu}(\sigma)}{d \sigma}\right|_{\sigma=0, \pi}=0 .
$$

Therefore, they are expanded in term of the cosine modes:

$$
\begin{aligned}
X^{\mu}(\sigma) & =\widehat{x}^{\mu}+i \sum_{n=1}^{\infty}\left(a_{n}-a_{n}^{\dagger}\right)^{\mu} \xi_{n}(\sigma), \\
P_{\mu}(\sigma) & =\frac{1}{\pi}\left[\widehat{p}_{\mu}+\sum_{n=1}^{\infty}\left(a_{n}+a_{n}^{\dagger}\right)_{\mu} \pi_{n}(\sigma)\right],
\end{aligned}
$$

where $\xi_{n}(\sigma)$ and $\pi_{n}(\sigma)$ are defined by

$$
\begin{aligned}
& \xi_{n}(\sigma)=\sqrt{\frac{2}{n}} \cos n \sigma, \\
& \pi_{n}(\sigma)=\sqrt{\frac{n}{2}} \cos n \sigma,
\end{aligned}
$$


and $(\widehat{x}, \widehat{p})$ and $\left(a_{n}, a_{n}^{\dagger}\right)$ satisfy

$$
\left[\widehat{x}^{\mu}, \widehat{p}_{\nu}\right]=i \delta_{\nu}^{\mu}, \quad\left[a_{m}^{\mu}, a_{n}^{\nu \dagger}\right]=\delta_{m, n} \eta^{\mu \nu}
$$

On the other hand, in open SFT in the background of lower dimensional D-branes, the string coordinates $X^{\perp}(\sigma)$ perpendicular to the D-brane should obey the Dirichlet BC:

$$
X^{\perp}(\sigma=0, \pi)=a^{\perp}
$$

where $a^{\perp}$ denotes the perpendicular coordinates of the brane. However, the two boundary conditions (1.1) and (1.7) are inconsistent if the two open string fields $\Phi^{(25)}$ and $\Phi^{(p)}$ describing the fluctuations around a D25-brane and a D $p$-brane with $p<25$ are related through the shift $\Phi^{(25)}=\Phi_{C}+\Phi^{(p)}$ using the D $p$-brane classical solution $\Phi_{C}$ in the SFT around the D25-brane.

One way to resolve this puzzle is to start with the open SFT of Witten [3] describing the D25-brane background and study the SFT obtained by reexpanding around the classical lump solution representing $\mathrm{D} p$-branes with $p<25$. Although the classical lump solutions in the open SFT have been obtained in the level truncation approximation to give expected results for their energy density [4, 5, 6], it seems hard to give a definite answer to our question of the change of the boundary conditions within this approximation. In this paper we shall adopt another way: we start with vacuum string field theory (VSFT) [7, 8], which is a candidate SFT expanded around the tachyon vacuum without any D-branes. The space-time open string coordinate $X^{\mu}(\sigma)$ as an argument of the string field of VSFT and its conjugate $P_{\mu}(\sigma)$ are subject to the Neumann BC and have expansions (1.2) and (1.3) since VSFT should describe the translationally invariant tachyon vacuum. What is good about VSFT is that exact lump solutions have been constructed [8, 9, 10]. Therefore, it is expected that we can carry out exact analyses on how the boundary condition switches to the Dirichlet one for the string coordinates perpendicular to these lumps.

Then, in what sense can the boundary condition change from the Neumann one to the Dirichlet one in the lump solution background of VSFT? The most conservative and modest test is whether the physical excitation spectrum around a lump solution agrees with that on the D-brane. Here we would like to pursue another possibility: $\left(X^{\perp}(\sigma), P_{\perp}(\sigma)\right)$ acting on any fluctuation modes around a lump solution satisfy the Dirichlet BC due to some singular nature of the solution [11, 12, 13]. Recall that the matter part $\Psi_{C}$ of the $p$-dimensional lump solution in VSFT satisfying the projector condition

$$
\Psi_{C} * \Psi_{C}=\Psi_{C}
$$

is factorized into the direct product of the projectors in each space-time direction:

$$
\left|\Psi_{C}\right\rangle=|N\rangle^{0} \otimes \cdots \otimes|N\rangle^{p} \otimes|D\rangle^{p+1} \otimes \cdots \otimes|D\rangle^{25}
$$


where $|N\rangle^{\mu}$ and $|D\rangle^{\mu}$ are the translationally invariant and the lump projectors for the direction $\mu$. And the tachyon fluctuation mode around $\Psi_{C}$ with center-of-mass momentum $k_{\|}$in the directions $\mu=0,1, \cdots, p$ parallel to the brane is given by injecting $k_{\|}$to the midpoint of $\Psi_{C} ; \exp \left(i k_{\|} X^{\|}(\pi / 2)\right)\left|\Psi_{C}\right\rangle$ [14, 15] (higher excitation modes in the longitudinal directions are given by multiplying the tachyon mode by suitable combinations of the creation operators $a_{n}^{\| \dagger}$ [16]). Therefore, in order for the change of the boundary conditions in the sense mentioned above is realized, it is at least necessary that the string coordinate $X^{\mu}(\sigma)$ acting on the lump projector $|D\rangle^{\mu}$ in the $\mu$-th direction vanishes at the endpoints (we are assuming that the lump is located at $\left.x^{\mu}=0\right)$ :

$$
X^{\mu}(\sigma=0, \pi)|D\rangle^{\mu}=0 .
$$

Besides the string coordinate $X^{\mu}(\sigma)$, its conjugate momentum $P_{\mu}(\sigma)$ should also satisfy the Dirichlet $\mathrm{BC}$ on $|D\rangle^{\mu}$. However, we must be careful about the string parameter $\sigma$. It may happen that $P_{\mu}(\sigma)|D\rangle^{\mu}$ diverges at the endpoints $\sigma=0$ and $\pi$ although the Dirichlet BC (1.10) for $X^{\mu}(\sigma)$ is realized. In this case we must suitably choose a new string parameter $s=s(\sigma)$ in the neighborhood of each of the endpoints in such a way that both $X^{\mu}(\sigma(s))|D\rangle^{\mu}$ and $P_{\mu}(s)|D\rangle^{\mu}$ have a regular series expansion in powers of $s$ (we assume that $s=0$ corresponds to the endpoint). Here, $P_{\mu}(s)$ with an argument $s$ denotes the conjugate momentum associated with the new string parameter; $P_{\mu}(s)=(d \sigma / d s) P_{\mu}(\sigma(s))$. We should examine whether the Dirichlet $\mathrm{BC}$ for the new conjugate momentum

$$
P_{\mu}(s=0)|D\rangle^{\mu}=0,
$$

is satisfied or not.

We carry out the test of the Dirichlet BC (1.10) and (1.11) on the one-parameter family of lump solutions given in [9, 10] using the boundary CFT technique. We find that the condition (1.10) for $X^{\mu}$ is satisfied only at a special value of the parameter. The condition (1.11) for the conjugate $P_{\mu}$, however, cannot be satisfied even at that value of the parameter. The same test of the Dirichlet BC is done also for another one-parameter family of lump projector obtained in [8] by the algebraic technique to lead to a negative result. Therefore, our conclusion is that the Dirichlet BC cannot be completely satisfied in the sense of (1.10) and (1.11) at lease for the known lump solutions in VSFT. As a byproduct of our analysis of the boundary conditions, we find that, contrary to the previous expectation, the one-parameter family of lump solutions given in [9, 10] and that given in [8] are different ones.

On the other hand, it has been known that the matter projectors have peculiar properties at the string midpoint $\sigma=\pi / 2$ : for example, we have $X^{\mu}(\pi / 2)|D\rangle^{\mu}=0$ for the lump projector [11].* This suggests that the midpoint could be interpreted as a kind of string

\footnotetext{
*See also [17, 18, 19, 20] for issues concerning the midpoint in VSFT.
} 
endpoint. We examine the midpoint behavior of the string coordinate and its conjugate acting on the projectors to find that the Dirichlet (Neumann) condition in the above sense is indeed satisfied for the lump (translationally invariant) projectors. Physical interpretation of this result, however, is still unclear.

In the above analyses of the boundary and the midpoint behaviors, we implicitly assume that the original oscillators $\left(a_{n}, a_{n}^{\dagger}\right)$ of (1.2) and (1.3) are the fundamental ones and study the behavior of their coefficient functions. However, we can define a new set of oscillators which is different from $\left(a_{n}, a_{n}^{\dagger}\right)$ and in a sense more natural in discussing the behaviors of the string coordinates and their conjugates acting on projectors. The new annihilation operators are defined to annihilate a projector instead of the Fock vacuum of the string modes. We examine how the boundary and the midpoint behaviors are changed if we adopt the new set of oscillators as the fundamental ones.

The organization of the rest of this paper is as follows. In sec. 2] we carry out the analysis of the boundary and the midpoint behaviors of the string coordinate and its conjugate acting on a lump projector. In sec. 3, we examine the midpoint behavior of the translationally invariant projector. In sec. 4, we discuss the disagreement of the two kinds of lump projectors by making use of the boundary conditions. Then, in sec. 5. we repeat the analysis of the midpoint behavior for the translationally invariant projector by taking the new oscillators. Finally in sec. 6] we summarize the paper and discuss further problems. In the appendices, various technical details used in the text are explained.

\section{Lump projector}

As stated in sec. 1, we would like to study the boundary and the midpoint behaviors of the string coordinate $X(\sigma)$ and its conjugate $P(\sigma)$ acting on the VSFT lump projectors. As a concrete example, let us first consider the lump solution proposed in [9] using the boundary CFT technique. Its explicit expression in the oscillator representation is given up to the overall normalization by 10]

$$
\left|D_{t}\right\rangle=\int_{-\infty}^{\infty} d p\left|\widetilde{D}_{t}(p)\right\rangle
$$

with

$$
\left|\widetilde{D}_{t}(p)\right\rangle=\exp \left(-\frac{1}{2} \sum_{m, n=1}^{\infty} Q_{m n} a_{m}^{\dagger} a_{n}^{\dagger}+p \sum_{n=1}^{\infty} \ell_{n} a_{n}^{\dagger}-\frac{1}{2} \lambda p^{2}\right)|p\rangle .
$$

Here and in the following, we omit the Lorentz index $\mu$ associated with the projector and the oscillators in it. In (2.2), $|p\rangle$ is the eigenstate of the center-of-mass momentum $\widehat{p}$ satisfying

$$
\widehat{p}|p\rangle=p|p\rangle, \quad \widehat{x}|p\rangle=-i \frac{\partial}{\partial p}|p\rangle,
$$


and $Q_{m n}, \ell_{n}$ and $\lambda$ are given as follows:

$$
\begin{aligned}
Q_{m n} & =\frac{1}{\sqrt{m n}} \oint_{z=0} \frac{d z}{2 \pi i} \frac{h^{\prime}(z)}{z^{m}} \oint_{w=0} \frac{d w}{2 \pi i} \frac{h^{\prime}(w)}{w^{n}} \frac{1}{(h(z)-h(w))^{2}} \frac{h(z) h(w)-t^{2}}{\sqrt{\left(t^{2}-h(z)^{2}\right)\left(t^{2}-h(w)^{2}\right)}} \\
\ell_{n} & =\sqrt{\frac{2}{n}} \oint_{z=0} \frac{d z}{2 \pi i} \frac{h^{\prime}(z)}{z^{n}} \frac{t}{h(z) \sqrt{t^{2}-h(z)^{2}}} \\
\lambda & =2 \ln (2 t)
\end{aligned}
$$

where the function $h(z)$ and its derivative are

$$
h(z)=\arctan z=\frac{1}{2 i} \ln \frac{1+i z}{1-i z}, \quad h^{\prime}(z)=\frac{1}{1+z^{2}} .
$$

Note that the matrix $Q_{m n}$ and the vector $\ell_{n}$ are both twist-even ones satisfying $C Q C=Q$ and $C \boldsymbol{\ell}=\boldsymbol{\ell}$ with $C_{m n}=(-1)^{n} \delta_{m n}$ being the twist matrix. The present solution $\left|D_{t}\right\rangle$ carries a real parameter $t$ which specifies the position of the twist operators in its BCFT construction. ${ }^{\dagger}$ The value of $t$ is restricted to $t \geq \pi / 4$.

Now letting $X(\sigma)(1.2)$ and $P(\sigma)(1.3)$ act on the solution $\left|D_{t}\right\rangle$ to express the result without using the annihilation operators, we get

$$
\begin{aligned}
& X(\sigma)\left|D_{t}\right\rangle=i \int d p\left(A(\sigma) p-\sum_{n=1}^{\infty} J_{n}(\sigma) a_{n}^{\dagger}\right)\left|\widetilde{D}_{t}(p)\right\rangle, \\
& P(\sigma)\left|D_{t}\right\rangle=\frac{1}{\pi} \int d p\left(B(\sigma) p-\sum_{n=1}^{\infty} K_{n}(\sigma) a_{n}^{\dagger}\right)\left|\widetilde{D}_{t}(p)\right\rangle,
\end{aligned}
$$

where the coefficient functions $A(\sigma), J_{n}(\sigma), B(\sigma)$ and $K_{n}(\sigma)$ are

$$
\begin{aligned}
A(\sigma) & =\boldsymbol{\ell} \cdot \boldsymbol{\xi}(\sigma)-\lambda, \\
\boldsymbol{J}(\sigma) & =(Q+1) \boldsymbol{\xi}(\sigma)-\boldsymbol{\ell}, \\
B(\sigma) & =\boldsymbol{\ell} \cdot \boldsymbol{\pi}(\sigma)+1, \\
\boldsymbol{K}(\sigma) & =(Q-1) \boldsymbol{\pi}(\sigma) .
\end{aligned}
$$

Using the integral representations (2.4) and (2.5), we can carry out the infinite summations (i.e., the multiplications between matrices and vectors) in (2.10) $-(2.13)$ to find that they are expressed in terms of two kinds of functions, $C(\sigma)$ and $I_{n}(\sigma)$ :

$$
C(\sigma)=\frac{1}{2} \ln \frac{t-\sqrt{t^{2}-h\left(e^{i \sigma-\epsilon}\right)^{2}}}{t+\sqrt{t^{2}-h\left(e^{i \sigma-\epsilon}\right)^{2}}},
$$

\footnotetext{
${ }^{\dagger}$ Our parameter $t$ is equal to $t_{0}$ in 10 .
} 


$$
I_{n}(\sigma)=\frac{1}{\sqrt{2 n}} \oint_{z=0} \frac{d z}{2 \pi i} \frac{h^{\prime}(z)}{z^{n}} \frac{1}{h\left(e^{i \sigma-\epsilon}\right)-h(z)} \sqrt{\frac{t^{2}-h\left(e^{i \sigma-\epsilon}\right)^{2}}{t^{2}-h(z)^{2}}}
$$

where $\epsilon$ is a positive infinitesimal and its origin is the regularization factor $e^{-n \epsilon}$ multiplying $\xi_{n}(\sigma)$ and $\pi_{n}(\sigma)$. In fact, the following relations hold (see appendix $\mathrm{A}$ for their derivation):

$$
\begin{aligned}
A(\sigma) & =C(\sigma)+C(-\sigma)=2 \operatorname{Re} C(\sigma), \\
J_{n}(\sigma) & =I_{n}(\sigma)+I_{n}(-\sigma)=2 \operatorname{Re} I_{n}(\sigma), \\
B(\sigma) & =\frac{1}{2 i} \frac{d}{d \sigma}[C(\sigma)-C(-\sigma)]=\frac{d}{d \sigma} \operatorname{Im} C(\sigma), \\
K_{n}(\sigma) & =\frac{1}{2 i} \frac{d}{d \sigma}\left[I_{n}(\sigma)-I_{n}(-\sigma)\right]=\frac{d}{d \sigma} \operatorname{Im} I_{n}(\sigma) .
\end{aligned}
$$

Note that

$$
C(\sigma)^{*}=C(-\sigma), \quad I_{n}(\sigma)^{*}=I_{n}(-\sigma)
$$

Having finished the preparation, we shall proceed to the study of the boundary and the midpoint behaviors of (2.8) and (2.9). Concretely, we shall examine the behaviors of the functions $A(\sigma), J_{n}(\sigma), B(\sigma)$ and $K_{n}(\sigma)$ which are the coefficients of $p$ and $a_{n}^{\dagger}$. For the sake of the easiness of explanation, we shall first look at the midpoint behaviors.

\subsection{Midpoint behavior}

It has been known that the lump projectors $|D\rangle$ of VSFT share the property $X(\pi / 2)|D\rangle=0$ [1]. Although the implication of this property is still controversial, it suggests that the midpoint could be interpreted as the endpoint. Here we shall study this property in more detail for the present $\left|D_{t}\right\rangle$ (2.1) for which the explicit calculation is possible. In particular, we are interested in the behavior of both $X(\sigma)\left|D_{t}\right\rangle$ and $P(\sigma)\left|D_{t}\right\rangle$ near the midpoint.

First, it is easily seen from $h\left(e^{ \pm i \pi / 2}\right)= \pm i \infty$ that $C(\pi / 2)$ and $I_{n}(\pi / 2)$ are both pureimaginary and hence we have $A(\pi / 2)=J_{n}(\pi / 2)=0$, implying that

$$
X\left(\frac{\pi}{2}\right)\left|D_{t}\right\rangle=0
$$

For studying the behaviors near the midpoint, it is convenient to express $h\left(e^{ \pm i \sigma}\right)$ in terms of a new real parameter $s(>0)$ as

$$
h\left(e^{ \pm i \sigma}\right)= \begin{cases}\frac{\pi}{4} \pm \frac{i}{2 s}, & \left(0 \leq \sigma<\frac{\pi}{2}\right), \\ -\frac{\pi}{4} \pm \frac{i}{2 s}, & \left(\frac{\pi}{2}<\sigma \leq \pi\right) .\end{cases}
$$


The explicit relation between $\sigma$ and $s$ is

$$
\frac{1}{s}=\ln \frac{1+\sin \sigma}{|\cos \sigma|} \underset{\sigma \sim \frac{\pi}{2}}{\simeq} \ln \frac{2}{|(\pi / 2)-\sigma|},
$$

and the point $s=0$ correspond to the midpoint $\sigma=\pi / 2$, while $s=\infty$ to the endpoints $\sigma=0$ and $\pi$. Then, it is easily seen that both $C(\sigma)$ (2.14) and $I_{n}(\sigma)(2.15)$ have regular series expansions in powers of $s$ around $s=0$, and that their real (imaginary) parts are odd (even) functions of $s$. For example, we have

$$
C(\sigma(s))=\frac{i \pi}{2}-2 t s-i \epsilon\left(\frac{\pi}{2}-\sigma\right) \pi t s^{2}+\left(\frac{\pi^{2}}{2}+\frac{4}{3} t^{2}\right) t s^{3}+O\left(s^{4}\right),
$$

where $\epsilon(x)=1(-1)$ when $x>0(x<0)$. Therefore, $X(\sigma(s))\left|D_{t}\right\rangle$ has an expansion in odd powers of $s$, which is consistent with the Dirichlet condition (2.21). Symbolically we have

$$
X(\sigma(s))\left|D_{t}\right\rangle=s+s^{3}+s^{5}+\ldots
$$

This implies that the natural string parameter near the midpoint is not the original one $\sigma$ but rather is $s$. Therefore, as the momentum variable conjugate to $X(s) \equiv X(\sigma(s))$ we should take $P(s)$ which is related to the original $P(\sigma)$ by ${ }^{\ddagger}$

$$
P(s)=\frac{d \sigma}{d s} P(\sigma),
$$

in order to satisfy

$$
\left[X(s), P\left(s^{\prime}\right)\right]=i \delta\left(s-s^{\prime}\right) .
$$

For this $P(s), P(s)\left|D_{t}\right\rangle$ is given by (2.9) with $B(\sigma)$ and $K_{n}(\sigma)$ replaced with

$$
B(s)=\frac{d \sigma}{d s} B(\sigma)=\frac{d}{d s} \operatorname{Im} C(\sigma), \quad K_{n}(s)=\frac{d \sigma}{d s} K_{n}(\sigma)=\frac{d}{d s} \operatorname{Im} I_{n}(\sigma),
$$

respectively. Recalling that $\operatorname{Im} C(\sigma)$ and $\operatorname{Im} I_{n}(\sigma)$ are even in $s$, we have symbolically

$$
P(s)\left|D_{t}\right\rangle=s+s^{3}+s^{5}+\ldots
$$

Namely, $P(s)\left|D_{t}\right\rangle$ also satisfies the Dirichlet condition at the midpoint. However, $P(\sigma)\left|D_{t}\right\rangle$ with the original string parameter $\sigma$ is divergent at the midpoint. This is seen from

$$
\frac{d s}{d \sigma}=-\frac{s^{2}}{\cos \sigma} \underset{s \sim 0}{\simeq}-\frac{1}{2} s^{2} e^{1 / s} \rightarrow \infty,
$$

obtained from (2.23). We shall discuss the implications of our result on the midpoint behaviors in sec. 6.

\footnotetext{
${ }^{\ddagger}$ We use the same symbol for the operators related by a conformal transformation and distinguish them by their arguments.
} 


\subsection{Endpoint behavior}

Next let us study the behaviors of $X(\sigma)\left|D_{t}\right\rangle$ and $P(\sigma)\left|D_{t}\right\rangle$ near the endpoints $\sigma=0$, $\pi$, which are directly related to the change of the boundary conditions (in the following we consider $\sigma=0)$. The endpoint behaviors of the basic functions $C(\sigma)(2.14)$ and $I_{n}(\sigma)(2.15)$ are completely different between the cases of $t>\pi / 4$ and $t=\pi / 4$ since we have $h(1)=\pi / 4: C(\sigma)$ and $I_{n}(\sigma)$ have regular Taylor expansions in powers of $\sigma$ for $t>\pi / 4$, while their expansions are in odd powers of $\sqrt{\sigma}$ (in particular, we have $C(0)=I_{n}(0)=0$ ) when $t=\pi / 4$. Explicitly, using the expansion

$$
h\left(e^{i \sigma}\right)=\frac{\pi}{4}+i\left(\frac{\sigma}{2}+\frac{\sigma^{3}}{12}+O\left(\sigma^{5}\right)\right)
$$

we have

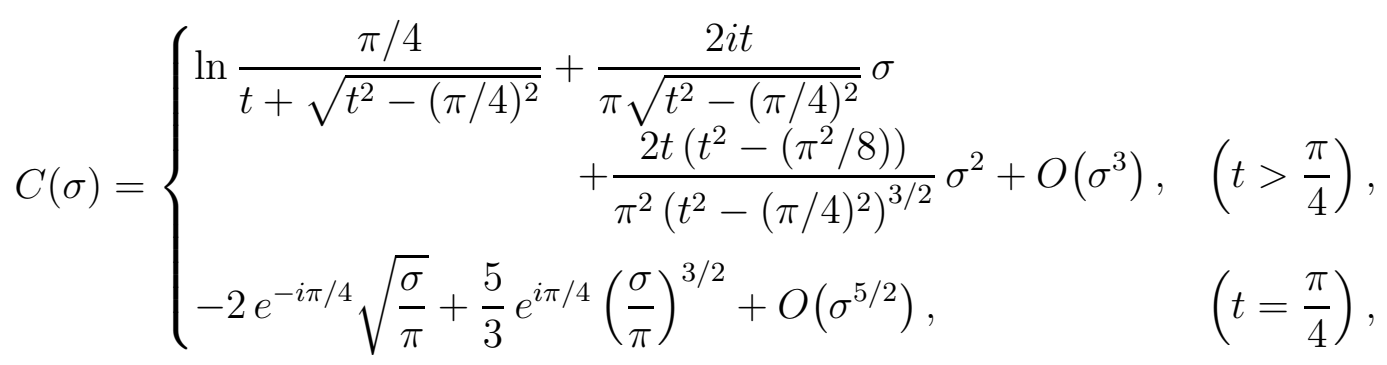

and

$$
\begin{aligned}
& I_{n}(\sigma)=\frac{1}{\sqrt{2 n}} \oint_{z=0} \frac{d z}{2 \pi i} \frac{h^{\prime}(z)}{z^{n}} \frac{1}{(\pi / 4)-h(z)} \\
& \quad \begin{cases}\sqrt{\frac{t^{2}-(\pi / 4)^{2}}{t^{2}-h(z)^{2}}}\left\{1-\frac{i\left(t^{2}-(\pi / 4) h(z)\right)}{2\left(t^{2}-(\pi / 4)^{2}\right)((\pi / 4)-h(z))} \sigma+O\left(\sigma^{2}\right)\right\}, & \left(t>\frac{\pi}{4}\right), \\
\frac{1}{\sqrt{(\pi / 4)^{2}-h(z)^{2}}}\left\{\frac{e^{-i \pi / 4}}{2} \sqrt{\pi \sigma}-\frac{e^{i \pi / 4}}{4 \pi^{2}} \frac{(3 \pi / 4)+h(z)}{(\pi / 4)-h(z)}(\pi \sigma)^{3 / 2}+O\left(\sigma^{5 / 2}\right)\right\}, & \left(t=\frac{\pi}{4}\right) .\end{cases}
\end{aligned}
$$

This result implies that, for $t>\pi / 4$, the original Neumann $\mathrm{BC}$ of $X(\sigma)$ and $P(\sigma)$ is kept unchanged even if they act on $\left|D_{t}\right\rangle$. However, the boundary condition is changed when these coordinates act on $\left|D_{\pi / 4}\right\rangle$ at the special value $t=\pi / 4$ which corresponds to putting the twist operators at ends of the local coordinate in the BCFT construction of the solution [9, 10]. Let us concentrate on the latter case in the rest of this subsection. First, $X(\sigma)\left|D_{\pi / 4}\right\rangle$ satisfies the Dirichlet $\mathrm{BC}$ at the endpoints:

$$
X(0)\left|D_{\pi / 4}\right\rangle=0
$$

Next, for considering the boundary condition of the conjugate momentum $P$, we have to specify the natural string parameter near the endpoints as we did for the midpoint in the previous subsection. Since (2.32) and (2.33) for $t=\pi / 4$ are expanded in odd powers of $\sqrt{\sigma}$, 
the natural string parameter near the endpoint $\sigma=0$ is $u=\sqrt{\sigma}$ rather than the original $\sigma$. For $X(u) \equiv X(\sigma)$ we have symbolically

$$
X(u)\left|D_{\pi / 4}\right\rangle=u+u^{3}+u^{5}+\ldots
$$

However, the expansion of $P(u)=(d \sigma / d u) P(\sigma)$ acting on $\left|D_{\pi / 4}\right\rangle$ is in even powers of $u$ :

$$
P(u)\left|D_{\pi / 4}\right\rangle=1+u^{2}+u^{4}+\ldots
$$

Therefore, even at the special point $t=\pi / 4$, the boundary condition of $P(u)\left|D_{\pi / 4}\right\rangle$ remains Neumann although that of $X(u)\left|D_{\pi / 4}\right\rangle$ is changed to Dirichlet. The change of the boundary conditions in the background of the lump solution (2.1) cannot be completely realized in the expected manner even in the case $t=\pi / 4$.

\section{Midpoint behavior of the Neumann projector}

We have seen in sec. 2.1 that the string coordinate and its conjugate momentum acting on the lump projector (2.1) perfectly satisfy the Dirichlet condition at the midpoint. In this section, we shall carry out the same kind of midpoint analysis for the translationally invariant Neumann projector $|N\rangle$ of VSFT [8], which is given up to normalization by

$$
|N\rangle=\exp \left(-\frac{1}{2} \sum_{m, n=1}^{\infty} S_{m n} a_{m}^{\dagger} a_{n}^{\dagger}\right)|p=0\rangle
$$

where the matrix $S_{m n}$ has the following integral representation:

$$
S_{m n}=\frac{1}{\sqrt{m n}} \oint_{z=0} \frac{d z}{2 \pi i} \frac{h^{\prime}(z)}{z^{m}} \oint_{w=0} \frac{d w}{2 \pi i} \frac{h^{\prime}(w)}{w^{n}} \frac{1}{(h(z)-h(w))^{2}} .
$$

Since $S_{m n}$ is equal to the $t \rightarrow \infty$ limit of $Q_{m n}$ (2.4) for the lump projector, i.e., $S_{m n}=$ $\lim _{t \rightarrow \infty} Q_{m n}$, most of the necessary formulas are obtained by simply taking the limit in the corresponding equations in sec. 2 for the lump projector. We have

$$
\begin{aligned}
& X(\sigma)|N\rangle=\left(\widehat{x}-i \sum_{n=1}^{\infty} J_{n}^{N}(\sigma) a_{n}^{\dagger}\right)|N\rangle, \\
& P(\sigma)|N\rangle=-\frac{1}{\pi} \sum_{n=1}^{\infty} K_{n}^{N}(\sigma) a_{n}^{\dagger}|N\rangle,
\end{aligned}
$$

where $J_{n}^{N}(\sigma)$ and $K_{n}^{N}(\sigma)$ are given by

$$
\boldsymbol{J}^{N}(\sigma)=(S+1) \boldsymbol{\xi}(\sigma)=\lim _{t \rightarrow \infty}(\boldsymbol{J}(\sigma)+\boldsymbol{\ell}),
$$




$$
\boldsymbol{K}^{N}(\sigma)=(S-1) \boldsymbol{\pi}(\sigma)=\lim _{t \rightarrow \infty} \boldsymbol{K}(\sigma)
$$

Taking the $t \rightarrow \infty$ limit in (2.17) and (2.19), we obtain

$$
\begin{aligned}
J_{n}^{N}(\sigma) & =2 \operatorname{Re} I_{n}^{N}(\sigma)-\sqrt{2} t_{n}, \\
K_{n}^{N}(\sigma) & =\frac{d}{d \sigma} \operatorname{Im} I_{n}^{N}(\sigma),
\end{aligned}
$$

where $I_{n}^{N}(\sigma)$ given by

$$
I_{n}^{N}(\sigma)=\lim _{t \rightarrow \infty} I_{n}(\sigma)=\frac{1}{\sqrt{2 n}} \oint_{z=0} \frac{d z}{2 \pi i} \frac{h^{\prime}(z)}{z^{n}} \frac{1}{h\left(e^{i \sigma-\epsilon}\right)-h(z)},
$$

and $t_{n}$ is the vector appearing in the fluctuation modes around the solution $|N\rangle[14$ :

$$
\lim _{t \rightarrow \infty} \ell_{n}=\sqrt{\frac{2}{n}} \oint_{z=0} \frac{d z}{2 \pi i} \frac{h^{\prime}(z)}{z^{n} h(z)}=-\sqrt{2} t_{n}
$$

The natural string parameter in the present case is again $s$ of (2.22). However, contrary to the case of $I_{n}(\sigma)$, the present $I_{n}^{N}(\sigma)$ without $\sqrt{\left(t^{2}-h\left(e^{i \sigma}\right)^{2}\right) /\left(t^{2}-h(z)^{2}\right)}$ has the property that its real (imaginary) part is even (odd) in $s$. Therefore, corresponding to (2.25) and (2.29), we have

$$
\begin{aligned}
& X(s)|N\rangle=1+s^{2}+s^{4}+\ldots, \\
& P(s)|N\rangle=1+s^{2}+s^{4}+\ldots
\end{aligned}
$$

Namely, both $X(s)|N\rangle$ and $P(s)|N\rangle$ are subject to the Neumann condition at the midpoint with respect to the new parameter $s$ :

$$
\left.\frac{d}{d s} X(s)|N\rangle\right|_{s=0}=\left.\frac{d}{d s} P(s)|N\rangle\right|_{s=0}=0 .
$$

As for the boundary conditions of $(X(\sigma), P(\sigma))|N\rangle$ at the endpoints $\sigma=0$ and $\pi$, they remain Neumann since nothing singular happens at the endpoints for the Neumann projector $|N\rangle$.

\section{Another lump projector}

Besides the lump projector $\left|D_{t}\right\rangle$ which we discussed in sec. 2, there is another kind of lump projector constructed in [8]. In this section we shall discuss the relationship between the two lump projectors by making use of their boundary behaviors. 
First, let us summarize the lump solution of [8]. In its construction, they introduced the zero-mode oscillator $\left(a_{0}, a_{0}^{\dagger}\right)$ which satisfies the usual commutation relation $\left[a_{0}, a_{0}^{\dagger}\right]=1$ :

$$
a_{0}=\frac{\sqrt{b}}{2} \widehat{p}-\frac{i}{\sqrt{b}} \widehat{x}, \quad a_{0}^{\dagger}=\frac{\sqrt{b}}{2} \widehat{p}+\frac{i}{\sqrt{b}} \widehat{x},
$$

where $b$ is an arbitrary positive parameter. The lump projector $\left|\Xi_{b}\right\rangle$ of $[8]$ is given up to normalization by

$$
\left|\Xi_{b}\right\rangle=\exp \left(-\frac{1}{2} \sum_{m, n=0}^{\infty} S_{m n}^{\prime} a_{m}^{\dagger} a_{n}^{\dagger}\right)\left|\Omega_{b}\right\rangle,
$$

where $\left|\Omega_{b}\right\rangle$ is the state annihilated by all $a_{n}$ including $a_{0}$ :

$$
a_{n}\left|\Omega_{b}\right\rangle=0, \quad(n \geq 0) .
$$

The coefficient matrix $S^{\prime}$ in the exponent is given in quite a similar manner to the algebraic construction of the translationally invariant solution (3.1) 21, 8]. We have to add a prime to all the matrices indicating that they are extended ones including $n=0$. First, $S^{\prime}$ is related to another matrix $T^{\prime}$ by

$$
S^{\prime}=C^{\prime} T^{\prime}
$$

with the (extended) twist-matrix $C_{m n}^{\prime}=\delta_{m n}(-1)^{n}(m, n \geq 0)$, and $T^{\prime}$ is given in terms of the primed Neumann matrix $M_{0}^{\prime}$ by

$$
T^{\prime}=\frac{1}{2 M_{0}^{\prime}}\left(1+M_{0}^{\prime}-\sqrt{\left(1-M_{0}^{\prime}\right)\left(1+3 M_{0}^{\prime}\right)}\right) .
$$

$M_{0}^{\prime}$ is expressed in terms of the unprimed Neumann matrix $M_{0}$ and the vectors $\boldsymbol{v}_{0}$ and $\boldsymbol{v}_{1}$ by

$$
M_{0}^{\prime}=\left(\begin{array}{cc}
\left(M_{0}^{\prime}\right)_{00} & \left(M_{0}^{\prime}\right)_{0 n} \\
\left(M_{0}^{\prime}\right)_{m 0} & \left(M_{0}^{\prime}\right)_{m n}
\end{array}\right)=\left(\begin{array}{cc}
1-\frac{2}{3} \frac{b}{\beta} & \frac{\sqrt{2 b}}{\beta} \boldsymbol{v}_{0}^{\mathrm{T}} \\
\frac{\sqrt{2 b}}{\beta} \boldsymbol{v}_{0} & M_{0}+\frac{1}{\beta}\left(-3 \boldsymbol{v}_{0} \boldsymbol{v}_{0}^{\mathrm{T}}+\boldsymbol{v}_{1} \boldsymbol{v}_{1}^{\mathrm{T}}\right)
\end{array}\right),
$$

where $\beta$ is

$$
\beta=2 V_{00}+\frac{b}{2}
$$

with

$$
V_{00}=\frac{1}{2} \ln \left(\frac{3^{3}}{2^{4}}\right) .
$$

The solution (4.2) has a free parameter $b$. It was conjectured that $b$ is a kind of gauge parameter [8] although no proof has been given yet.

\footnotetext{
${ }^{\S}$ Our convention of the Neumann matrices and vectors is given in [13].
} 
Using the relation

$$
|p\rangle=\left(\frac{2 \pi}{b}\right)^{-1 / 4} \exp \left(-\frac{b}{4} p^{2}+\sqrt{b} p a_{0}^{\dagger}-\frac{1}{2}\left(a_{0}^{\dagger}\right)^{2}\right)\left|\Omega_{b}\right\rangle,
$$

the lump projector (2.1) we considered in sec. 2 can also be put into the squeezed state form (4.2) using the zero-mode oscillators. The corresponding $S^{\prime}$ is

$$
\begin{aligned}
& S_{m n}^{\prime}=Q_{m n}-\frac{\ell_{m} \ell_{n}}{\lambda+(b / 2)}, \quad(m, n \geq 1), \\
& S_{n 0}^{\prime}=S_{0 n}^{\prime}=-\frac{\sqrt{b}}{\lambda+(b / 2)} \ell_{n}, \quad(n \geq 1), \\
& S_{00}^{\prime}=\frac{\lambda-(b / 2)}{\lambda+(b / 2)}
\end{aligned}
$$

Now let us proceed to discussing the relation between the lump solutions. It was conjectured in [10] that the two lump solutions, (2.1) with parameter $t$ and (4.2) with $b$, are the same thing; namely, they agree each other by giving $t$ as a function of $b$ (or vice versa). Numerical analysis for the relations (4.10)-(4.12) was carried out in [10] to obtain results supporting the conjecture. In this section, however, using the boundary conditions of $X(\sigma)$ acting on the two kinds of lump projectors, we present a negative result for the agreement of the two. More precisely, we shall show that the solution (2.1) at $t=\pi / 4$ cannot coincide with the other solution (4.2) for any value of the parameter $b . \uparrow$

First recall (2.34), namely, that $X(0)$ acting on the solution (2.1) at $t=\pi / 4$ vanishes. On the other hand, for the solution (4.2) we have

$$
X(\sigma)\left|\Xi_{b}\right\rangle=-i\left[\boldsymbol{a}^{\prime \dagger} \cdot\left(S^{\prime}+1\right) \boldsymbol{\xi}^{\prime}(\sigma)\right]\left|\Xi_{b}\right\rangle,
$$

where the primed vectors $\boldsymbol{\xi}^{\prime}(\sigma)$ and $\boldsymbol{a}^{\prime}$ are defined by

$$
\boldsymbol{\xi}^{\prime}(\sigma)=\left(\begin{array}{c}
\frac{\sqrt{b}}{2} \\
\sqrt{\frac{2}{n}} \cos n \sigma
\end{array}\right)=\left(\begin{array}{c}
\frac{\sqrt{b}}{2} \\
\boldsymbol{\xi}(\sigma)
\end{array}\right),
$$

and $\boldsymbol{a}^{\prime}=\left(a_{0}, a_{1}, a_{2}, \cdots\right)^{\mathrm{T}}$ including $a_{0}$, respectively. Therefore, the condition for

$$
X(0)\left|\Xi_{b}\right\rangle=0
$$

is $\left(S^{\prime}+1\right) \boldsymbol{\xi}^{\prime}(0)=0$, which is equivalent to

$$
\left(T^{\prime} \pm 1\right) \frac{1 \pm C^{\prime}}{2} \boldsymbol{\xi}^{\prime}(0)=0
$$

\footnotetext{
IFrom the (numerical) agreement of the two $S_{00}^{\prime}, S_{00}^{\prime}$ for (4.2) given by (4.5) and $S_{00}^{\prime}$ of (4.12), it was claimed in [10] that $t=\pi / 4$ corresponds to $b \simeq 1.16$.
} 
for the twist-even and odd parts of $\boldsymbol{\xi}^{\prime}(0)$. Since $T^{\prime}$ is given by (4.5) in terms of $M_{0}^{\prime}$, these conditions are translated into the following two:

$$
\begin{aligned}
\left(M_{0}^{\prime}+\frac{1}{3}\right) \frac{1+C^{\prime}}{2} \boldsymbol{\xi}^{\prime}(0) & =0, \\
\left(M_{0}^{\prime}-1\right) \frac{1-C^{\prime}}{2} \boldsymbol{\xi}^{\prime}(0) & =0 .
\end{aligned}
$$

Let us consider the first condition (4.17). Its LHS is given, using (4.6) and that $\boldsymbol{v}_{0}$ is twist-even, by

$$
\left(M_{0}^{\prime}+\frac{1}{3}\right) \frac{1+C^{\prime}}{2} \boldsymbol{\xi}^{\prime}(\sigma)=\left(\begin{array}{c}
\frac{\sqrt{2 b}}{\beta}\left(\boldsymbol{v}_{0} \cdot \boldsymbol{\xi}(\sigma)+\frac{2 \sqrt{2}}{3} V_{00}\right) \\
\frac{1}{\beta}\left(\frac{b}{\sqrt{2}}-3 \boldsymbol{v}_{0} \cdot \boldsymbol{\xi}(\sigma)\right) \boldsymbol{v}_{0}+\left(M_{0}+\frac{1}{3}\right) \frac{1+C}{2} \boldsymbol{\xi}(\sigma)
\end{array}\right)
$$

and hence (4.17) is reduced to the following $b$-independent conditions for the unprimed quantities:

$$
\begin{aligned}
\boldsymbol{v}_{0} \cdot \boldsymbol{\xi}(\sigma) & =-\frac{2 \sqrt{2}}{3} V_{00}, \\
\left(M_{0}+\frac{1}{3}\right) \frac{1+C}{2} \boldsymbol{\xi}(\sigma) & =-\sqrt{2} \boldsymbol{v}_{0}
\end{aligned}
$$

However, the condition (4.20) can be shown to be invalid. In fact, the LHS of (4.20) is calculated using the integral representation of $\boldsymbol{v}_{0}$ to give

$$
\boldsymbol{v}_{0} \cdot \boldsymbol{\xi}(0)=-\frac{2 \sqrt{2}}{3} V_{00}+\frac{\sqrt{2}}{3} \ln 2 .
$$

A derivation of (4.22) is given in appendix A. Therefore, the boundary condition (4.15) cannot hold for any value of the parameter $b$, and hence $\left|D_{\pi / 4}\right\rangle$ at $t=\pi / 4$ cannot agree with $\left|\Xi_{b}\right\rangle$ for any $b . \|$

\section{New oscillators}

When we considered the boundary or midpoint behaviors of $(X(\sigma), P(\sigma))$ acting on the various projectors $\mid$ Proj $\rangle$, we studied the coefficient functions of $a_{n}^{\dagger}|\operatorname{Proj}\rangle$. However, there is a priori no reason why we have to look at the coefficients of $a_{n}^{\dagger}|\operatorname{Proj}\rangle$ with the original creation operator $a_{n}^{\dagger}$; in particular, $a_{n}^{\dagger}$ is not a finite operator on the projectors in the sense that the norm of $a_{n}^{\dagger}|\operatorname{Proj}\rangle$ is not finite even if $\mid$ Proj $\rangle$ is normalized.

\footnotetext{
"Eq. (2.21) at the midpoint holds also for $\left|\Xi_{b}\right\rangle$ since we have $\left(S^{\prime}+1\right) \boldsymbol{\xi}^{\prime}(\pi / 2)=0$ [11].
} 
In this subsection, we shall propose another way of considering the boundary or midpoint behaviors. This is to take, instead of the original $a_{n}$, the following $b_{n}$ :** $^{* *}$

$$
\boldsymbol{b}^{\prime}=\frac{1}{\sqrt{1-S^{\prime 2}}}\left(\boldsymbol{a}^{\prime}+S^{\prime} \boldsymbol{a}^{\prime \dagger}\right)
$$

with $\boldsymbol{b}^{\prime}=\left(b_{0}, b_{1}, b_{2}, \cdots\right)^{\mathrm{T}}$ including $b_{0}$. Here we are considering a lump projector $\left(|\operatorname{Proj}\rangle=\left|D_{t}\right\rangle\right.$ or $\left.\left|\Xi_{b}\right\rangle\right)$ of the form (4.2) in the $\left(a_{0}, a_{0}^{\dagger}\right)$ representation for the zero-mode. The basic properties of $b_{n}$ are that it annihilates the solution,

$$
b_{n}|\operatorname{Proj}\rangle=0, \quad(n=0,1,2, \cdots),
$$

and that it is normalized:

$$
\left[b_{m}, b_{n}^{\dagger}\right]=\delta_{m n} .
$$

In terms of the new oscillator $\left(b_{n}, b_{n}^{\dagger}\right)$, the string coordinate and its conjugate momentum are expanded as

$$
\begin{aligned}
& X(\sigma)=i\left(\boldsymbol{b}^{\prime}-\boldsymbol{b}^{\prime \dagger}\right) \cdot \boldsymbol{\Xi}^{\prime}(\sigma), \\
& P(\sigma)=\frac{1}{\pi}\left(\boldsymbol{b}^{\prime}+\boldsymbol{b}^{\prime \dagger}\right) \cdot \boldsymbol{\Pi}^{\prime}(\sigma),
\end{aligned}
$$

where the function sets $\boldsymbol{\Xi}^{\prime}(\sigma)=\left(\Xi_{n}^{\prime}(\sigma)\right)$ and $\boldsymbol{\Pi}^{\prime}(\sigma)=\left(\Pi_{n}^{\prime}(\sigma)\right)$ are defined by

$$
\begin{aligned}
& \boldsymbol{\Xi}^{\prime}(\sigma)=\left(\frac{1+S^{\prime}}{1-S^{\prime}}\right)^{1 / 2} \boldsymbol{\xi}^{\prime}(\sigma), \\
& \boldsymbol{\Pi}^{\prime}(\sigma)=\left(\frac{1-S^{\prime}}{1+S^{\prime}}\right)^{1 / 2} \boldsymbol{\pi}^{\prime}(\sigma),
\end{aligned}
$$

with $\boldsymbol{\xi}^{\prime}(\sigma)$ of (4.14) and

$$
\boldsymbol{\pi}^{\prime}(\sigma)=\left(\begin{array}{c}
\frac{1}{\sqrt{b}} \\
\sqrt{\frac{n}{2}} \cos n \sigma^{2}
\end{array}\right)=\left(\begin{array}{c}
\frac{1}{\sqrt{b}} \\
\boldsymbol{\pi}(\sigma)
\end{array}\right) .
$$

If we regard $\left(b_{n}, b_{n}^{\dagger}\right)$ as the basic oscillator, it is the behavior of $\boldsymbol{\Xi}^{\prime}(\sigma)$ and $\boldsymbol{\Pi}^{\prime}(\sigma)$ at the boundary or the midpoint that matters. However, this analysis is not an easy task for the lump solutions since there appear square roots of matrices in the definitions (5.6) and (5.7).

For the translationally invariant solution $|N\rangle$ (3.1), we can similarly define new oscillator $b_{n}$ annihilating $|N\rangle$. It is given by the unprimed version of (5.1). The corresponding mode functions are

$$
\boldsymbol{\Xi}(\sigma)=\left(\frac{1+S}{1-S}\right)^{1 / 2} \boldsymbol{\xi}(\sigma),
$$

** The change of oscillators from $\boldsymbol{a}^{\prime}$ to $\boldsymbol{b}^{\prime}$ was considered previously by [22] in the context of half-string formalism [17, 18, 19, 20]. We are grateful to the authors of [22] for informing us of their work. 


$$
\boldsymbol{\Pi}(\sigma)=\left(\frac{1-S}{1+S}\right)^{1 / 2} \boldsymbol{\pi}(\sigma)
$$

In this case of $|N\rangle$ we can carry out explicit calculations since the eigenvalue problem of the Neumann matrices has been solved completely [23]. We present the outline of the calculation in appendix $\mathbb{B}$. In the neighborhood of the midpoint $\sigma=\pi / 2, \Xi_{n}(\sigma)$ is expanded in odd powers of $\sqrt{s}$ with $s$ defined by (2.22):

$$
\Xi_{n}(\sigma)=\text { const. }+\sqrt{\frac{2}{n}} \oint_{z=0} \frac{d z}{2 \pi i} \frac{h^{\prime}(z)}{z^{n}}\left[\sqrt{s}-h(z) s^{3 / 2}+O\left(s^{5 / 2}\right)\right] .
$$

On the other hand, $\Pi_{n}(\sigma)$ is given as

$$
\Pi_{n}(\sigma)=\frac{d}{d \sigma} w_{n}(\sigma)
$$

with $w_{n}(\sigma)$ having also an expansion in odd powers of $\sqrt{s}$ :

$$
w_{n}(\sigma)=\frac{1}{\sqrt{2 n}} \oint_{z=0} \frac{d z}{2 \pi i} \frac{h^{\prime}(z)}{z^{n}}\left[\sqrt{s}+h(z) s^{3 / 2}+O\left(s^{5 / 2}\right)\right] .
$$

Recall that $I_{n}^{N}(\sigma)$ giving $\boldsymbol{J}^{N}(\sigma)$ (3.5) and $\boldsymbol{K}^{N}(\sigma)$ has an expansion in integer powers of $s$. In the present case the expansions have been changed to those in terms of half an odd integer powers of $s$. This is due to the square roots in (5.9) and (5.10) and that the matrix $S$ has the eigenvalue 1 (see appendix (B)).

Eq. (5.11) and (5.13) imply that the natural string parameter near the midpoint is $v=\sqrt{s}$ rather than $s$. The mode function $\Pi_{n}(v)$ for the momentum $P(v)$ corresponding to the string parameter $v$ is

$$
\Pi_{n}(v)=\frac{d}{d v} \operatorname{Im} w_{n}(\sigma)=\frac{1}{\sqrt{2 n}} \oint_{z=0} \frac{d z}{2 \pi i} \frac{h^{\prime}(z)}{z^{n}}\left[1+3 h(z) v^{2}+O\left(v^{4}\right)\right],
$$

and it is subject to the Neumann condition at the midpoint (namely, $(d / d v) \Pi_{n}(v)=0$ at $v=0)$. However, $\Xi_{n}(v)=\Xi_{n}(\sigma)$ (5.11) for the coordinate $X(v)$ has an expansion in odd powers of $v$ and does not satisfy the Neumann condition at the midpoint. Namely, the midpoint behavior of the string coordinate acting on $|N\rangle$ of (3.1) depends on the choice of the basic oscillators, $\left(a_{n}, a_{n}^{\dagger}\right)$ or $\left(b_{n}, b_{n}^{\dagger}\right)$. It is our future problem to carry out similar analysis of the boundary behavior for the lump projectors.

\section{Summary and discussions}

In this paper we studied the endpoint and the midpoint behaviors of the string coordinate and its conjugate momentum acting on the various matter projectors in VSFT. Our original 
expectation was that the Dirichlet $\mathrm{BC}$ is realized on the lump projectors in spite of the fact that the string coordinates are defined to obey the Neumann BC in VSFT. Our findings are summarized as follows:

- The endpoint and the midpoint behaviors of the lump projector $\left|D_{t}\right\rangle$ (2.1) with a parameter $t$ and those of the translationally invariant projector $|N\rangle$ (3.1) are summarized in table 1. Here, we adopted the original oscillators $\left(a_{n}, a_{n}^{\dagger}\right)$ as the basic ones; namely, we examined the behaviors of the coefficient functions of $a_{n}^{\dagger}$ in $(X, P)|\operatorname{Proj}\rangle$.

- The string coordinate acting on another lump projector $\left|\Xi_{b}\right\rangle$ (4.2) carrying a parameter $b$ does not satisfy the Dirichlet BC at the endpoints for any $b$. This is a negative result against the identification of the two lump projectors $\left|D_{t}\right\rangle$ and $\left|\Xi_{b}\right\rangle[10$.

- If, instead of the original oscillators $a_{n}$, we adopt as the basic oscillators the new set $b_{n}$ (5.1) which annihilates the projector, the Neumann property of $X(\sigma)|N\rangle$ at the midpoint no longer holds.

\begin{tabular}{|c|c|c|c|c|}
\hline \multirow{2}{*}{} & \multicolumn{2}{|c|}{ endpoint } & \multicolumn{2}{c|}{ midpoint } \\
\cline { 2 - 5 } & $X$ & $P$ & $X$ & $P$ \\
\hline$\left|D_{t=\pi / 4}\right\rangle$ & $\mathrm{D}$ & $\mathrm{N}$ & $\mathrm{D}$ & $\mathrm{D}$ \\
\hline$\left|D_{t>\pi / 4}\right\rangle$ & $\mathrm{N}$ & $\mathrm{N}$ & $\mathrm{D}$ & $\mathrm{D}$ \\
\hline$|N\rangle$ & $\mathrm{N}$ & $\mathrm{N}$ & $\mathrm{N}$ & $\mathrm{N}$ \\
\hline
\end{tabular}

Table 1: The endpoint and the midpoint behaviors of the string coordinate $X$ and its conjugate momentum $P$ acting on the projectors $\left|D_{t}\right\rangle$ and $|N\rangle$ of VSFT. N and D denote the Neumann and the Dirichlet conditions, respectively.

There remain many questions left unanswered. First, as seen from table 11 our original expectation on the dynamical change of the boundary conditions of $(X, P)$ from the Neumann to the Dirichlet on the lump background is not completely realized. Even in the case of $\left|D_{t}\right\rangle$ at $t=\pi / 4$, the conjugate momentum remains Neumann at the boundary although the string coordinate obeys the Dirichlet BC. This may imply that our expectation that change of the boundary conditions occurs in $(X, P) \mid$ Proj $\rangle$ is too strong. Another possibility would be that the change of the boundary conditions is realized in the coefficient functions of other set of creation operators in $(X, P)|\operatorname{Proj}\rangle$ than the original $a_{n}^{\dagger}$. A candidate of such new set oscillators is $\left(b_{n}, b_{n}^{\dagger}\right)$ of sec. 5. In this paper, we saw only that the midpoint behaviors of $(X, P)|N\rangle$ are different between the $a_{n}^{\dagger}$ and the $b_{n}^{\dagger}$ cases. Carrying out a complete analysis of the boundary conditions using $b_{n}^{\dagger}$ for the lump projectors $\left|D_{t}\right\rangle$ and $\left|\Xi_{b}\right\rangle$ is one of our future 
problems. Analysis of the eigenvalue problem of the primed Neumann matrices in [24 may be useful. It is interesting if the new set of oscillators have special meaning in the construction of the fluctuation modes around the solution in VSFT [14, 16].

Our second problem is the interpretation of the midpoint behaviors of $(X, P)|\operatorname{Proj}\rangle$. In this paper, we found that the midpoint obeys perfectly the Neumann and the Dirichlet condition in the cases of the translationally invariant and the lump projectors, respectively. This may merely be a manifestation of the singular nature of VSFT with purely ghost BRST operator [11, 25]. A more positive and radical interpretation of this phenomenon would be that the midpoint

is in fact the open string boundary. In particular for $|N\rangle$, the property $\int_{0}^{\pi / 2} d \sigma P(\sigma)|N\rangle=0$ implying naively that we can split the left and the right halves of the open string [11, 26] seems to support our expectation. Pursuing this possibility is also our interesting future problem.

\section{Acknowledgments}

We would like to thank H. Ooguri and S. Teraguchi for valuable discussions and comments. S. M. is also grateful to theoretical particle physics group of Department of Physics, Kyoto University for hospitality. The work of H. H. was supported in part by the Grant-in-Aid for Scientific Research (C) No. 15540268 from Japan Society for the Promotion of Science (JSPS). The work of S. M. was supported in part by the DOE grant DE-FG03-92-ER40701.

\section{Appendix}

\section{A Derivation of $(\underline{2.16})-(\underline{2.19})$ and $(4.22)$}

In this appendix we briefly summarize the points in deriving (2.16)-(2.19) and (4.22). In carrying out the infinite summations in (2.10) $-(2.13)$, we multiply $\xi_{n}(\sigma)$ and $\pi_{n}(\sigma)$ by the regularization factor $e^{-n \epsilon}$ and let $\epsilon \rightarrow+0$ in the end. Let us consider, for example, $J_{n}(\sigma)$ (2.11). For this it is convenient to use another expression of $Q_{m n}$,

$$
Q_{m n}=\sqrt{\frac{n}{m}} \oint_{z=0} \frac{d z}{2 \pi i} \frac{h^{\prime}(z)}{z^{m}} \oint_{w=0} \frac{d w}{2 \pi i} \frac{1}{w^{n+1}} \frac{1}{h(w)-h(z)} \sqrt{\frac{t^{2}-h(w)^{2}}{t^{2}-h(z)^{2}}},
$$

which is obtained from (2.4) by integration by parts. Then the first term on the RHS of (2.11) with the regularization is given by

$$
\sum_{n=1}^{\infty} Q_{m n} \xi_{n}(\sigma) e^{-n \epsilon}
$$




$$
=\sqrt{\frac{2}{m}} \oint_{z=0} \frac{d z}{2 \pi i} \frac{h^{\prime}(z)}{z^{m}} \oint_{C_{w}} \frac{d w}{2 \pi i} \frac{1}{h(w)-h(z)} \sqrt{\frac{t^{2}-h(w)^{2}}{t^{2}-h(z)^{2}}} \frac{1}{2 w} \sum_{ \pm} \frac{1}{w e^{\epsilon \pm i \sigma}-1} .
$$

The contour $C_{w}$ of the $w$-integration should be such that encloses $w=e^{ \pm i \sigma-\epsilon}$ but excludes the branch points $w= \pm i$ of $h(w)$. The former requirement is due to the convergence of the geometric series $\sum_{n=1}^{\infty} \cos n \sigma e^{-n \epsilon} / w^{n+1}$. Therefore, we should take residues at four points $w=0, z$, and $e^{-\epsilon \pm i \sigma}$ in carry out the $w$-integration (the contour of $z$-integration is a small one enclosing $z=0$ ), which give $\ell_{m},-\xi_{m}(\sigma)$ and $I_{m}( \pm \sigma)$, respectively, in the limit $\epsilon \rightarrow+0$. This finishes the proof of (2.17). Derivations of (2.18) and (2.19) are quite similar.

Next, the derivation of (2.16) is a bit more involved. The first term of $A(\sigma)$ (2.10) with the regularization factor is written as

$$
\sum_{n=1}^{\infty} \ell_{n} \xi_{n}(\sigma) e^{-n \epsilon}=\oint_{C_{z}} \frac{d z}{2 \pi i}\left(\frac{d}{d z} \ln U(z)-\frac{1}{z}\right) \sum_{ \pm} \ln \left(\frac{z-e^{ \pm i \sigma-\epsilon}}{z}\right)
$$

where the contour $C_{z}$ is the same one as $C_{w}$ for (A.2), and $U(z)$ is given by

$$
U(z)=\frac{z}{h(z)}\left(t+\sqrt{t^{2}-h(z)^{2}}\right) .
$$

Note that $U(z)$ is regular inside $C_{z}$, in particular, at $z=0$. By taking as $C_{z}$ the ones running just above and below the logarithmic cut connecting $z=0$ and $e^{ \pm i \sigma-\epsilon}$ for each of the two terms in the summation $\sum_{ \pm}$in (A.3), we see that the contribution to (A.3) of the $(d / d z) U(z)$ term is $\sum_{ \pm}\left(\ln U(0)-\ln U\left(e^{ \pm i \sigma}\right)\right)=\lambda+C(\sigma)+C(-\sigma)$, while that of the $-1 / z$ term vanishes. Hence we get (2.16).

Finally, we present the derivation of (4.22), which is quite similar to that of (2.16) explained above. For this we need the integration formula for the Neumann vector $\boldsymbol{v}_{0}$ :

$$
\left(\boldsymbol{v}_{0}\right)_{n}=-\frac{1}{3 \sqrt{n}} \oint_{z=0} \frac{d z}{2 \pi i} \frac{f^{\prime}(z)}{z^{n}}\left[\frac{2}{f(z)-1}-\frac{1}{f(z)-\omega}-\frac{1}{f(z)-\omega^{*}}\right]
$$

with

$$
f(z)=\left(\frac{1+i z}{1-i z}\right)^{2 / 3}, \quad f^{\prime}(z)=\frac{4 i}{3} \frac{f(z)}{1+z^{2}}, \quad \omega=e^{2 \pi i / 3} .
$$

Then, $\boldsymbol{v}_{0} \cdot \boldsymbol{\xi}(\sigma)$ with the regularization is calculated as follows:

$$
\begin{aligned}
\sum_{n=1}^{\infty}\left(\boldsymbol{v}_{0}\right)_{n} \xi_{n}(\sigma) e^{-n \epsilon} & =\frac{1}{3 \sqrt{2}} \oint_{C_{z}} \frac{d z}{2 \pi i}\left(\frac{d}{d z} \ln W(z)+\frac{2}{z}\right) \sum_{ \pm} \ln \left(\frac{z-e^{ \pm i \sigma-\epsilon}}{z}\right) \\
& =\frac{1}{3 \sqrt{2}} \sum_{ \pm} \ln \frac{W(0)}{W\left(e^{ \pm i \sigma}\right)}=-\frac{2 \sqrt{2}}{3} V_{00}-\frac{1}{3 \sqrt{2}} \sum_{ \pm} \ln W\left(e^{ \pm i \sigma}\right),
\end{aligned}
$$


where $W(z)$ is defined by

$$
W(z)=\frac{(f(z)-1)^{2}}{\left(-z^{2}\right)(f(z)-\omega)\left(f(z)-\omega^{*}\right)},
$$

and we have used that $W(0)=2^{4} / 3^{3}$. Putting $\sigma=0$ in A.7 and using that $W(1)=1 / 2$, we obtain (4.22).

\section{B $\quad \boldsymbol{\Xi}(\sigma)$ and $\Pi(\sigma)$}

In this appendix we derive the series expansions (5.11) and (5.13) of the functions $\boldsymbol{\Xi}(\sigma)$ (5.9) and $\Pi(\sigma)$ (5.10) near the midpoint. We apply the technique developed in [23] and [27, 28] for the eigenvalue problem of the Neumann coefficients of the translationally invariant solution. Here we use bras and kets for the vectors by following [27, 28]. Let $|\kappa\rangle$ be the eigenvector of the the infinite dimensional symmetric matrix $K_{1}$ with components $\left(K_{1}\right)_{m n}=$ $-\sqrt{(m-1) m} \delta_{m-1, n}-\sqrt{m(m+1)} \delta_{m+1, n}$ corresponding to the eigenvalue $\kappa$ :

$$
K_{1}|\kappa\rangle=\kappa|\kappa\rangle, \quad(-\infty<\kappa<\infty) .
$$

The Neumann matrix $M_{0}$ is expressed in terms of $K_{1}$ as $M_{0}=-\left(1+2 \cosh \left(\pi K_{1} / 2\right)\right)^{-1}$, and hence $|\kappa\rangle$ is also the eigenvector of $T=S C$ given by (4.5) without primes:

$$
T|\kappa\rangle=-e^{-(\pi / 2)|\kappa|}|\kappa\rangle
$$

Other basic formulas concerning $|\kappa\rangle$ are

$$
\begin{aligned}
\langle n \mid \kappa\rangle & =\frac{\sqrt{n}}{\kappa} \oint_{z=0} \frac{d z}{2 \pi i} \frac{1}{z^{n+1}}\left(1-e^{-\kappa h(z)}\right), \\
\int_{-\infty}^{\infty} \frac{d \kappa}{\mathcal{N}(\kappa)}|\kappa\rangle\langle\kappa| & =\mathbf{1}, \quad\left(\mathcal{N}(\kappa)=\frac{2}{\kappa} \sinh \frac{\pi \kappa}{2}\right) \\
C|\kappa\rangle & =-|-\kappa\rangle
\end{aligned}
$$

where $|n\rangle(n=1,2,3, \cdots)$ is the vector with its $n$-th component equal to one and all other components equal to zero.

The inner-products of $|\kappa\rangle$ with the vectors $|\xi(\sigma)\rangle$ (1.4) and $|\pi(\sigma)\rangle$ (1.5) are calculated using (B.3) to give

$$
\begin{aligned}
\langle\kappa \mid \xi(\sigma)\rangle & =\frac{\sqrt{2}}{\kappa} \operatorname{Re}\left(1-e^{-\kappa h\left(e^{i \sigma}\right)}\right), \\
\langle\kappa \mid y(\sigma)\rangle & =\frac{1}{\sqrt{2} \kappa} \frac{d}{d \sigma} \operatorname{Im}\left(1-e^{-\kappa h\left(e^{i \sigma}\right)}\right) .
\end{aligned}
$$


Then, using the above formulas and

$$
\left(\frac{1 \pm S}{1 \mp S}\right)^{1 / 2}|\kappa\rangle=\frac{1 \pm T C}{\sqrt{1-T^{2}}}|\kappa\rangle=\frac{1}{\sqrt{1-e^{-\pi|\kappa|}}}\left(|\kappa\rangle \pm e^{-(\pi / 2)|\kappa|}|-\kappa\rangle\right)
$$

we obtain

$$
\begin{aligned}
\Xi_{n}(\sigma) & =\int_{-\infty}^{\infty} \frac{d \kappa}{\mathcal{N}(\kappa)}\left\langle n\left|\left(\frac{1+S}{1-S}\right)^{1 / 2}\right| \kappa\right\rangle\langle\kappa \mid \xi(\sigma)\rangle \\
& =\sqrt{\frac{2}{n}} \operatorname{Re} \oint_{z=0} \frac{d z}{2 \pi i} \frac{h^{\prime}(z)}{z^{n}}\left\{L_{1}(h(z))-L_{2}(h(z))-L_{1}\left(h\left(e^{i \sigma}\right)+h(z)\right)-L_{2}\left(h\left(e^{i \sigma}\right)-h(z)\right)\right\},
\end{aligned}
$$

where $L_{1}(h)$ and $L_{2}(h)$ are defined by

$$
\begin{aligned}
& L_{1}(h)=\int_{0}^{\infty} \frac{d \kappa}{\kappa \mathcal{N}(\kappa)} \frac{1}{\sqrt{1-e^{-\pi \kappa}}}\left(e^{-\kappa h}-e^{\kappa h}\right), \\
& L_{2}(h)=\int_{0}^{\infty} \frac{d \kappa}{\kappa \mathcal{N}(\kappa)} \frac{e^{-(\pi / 2) \kappa}}{\sqrt{1-e^{-\pi \kappa}}}\left(e^{-\kappa h}-e^{\kappa h}\right) .
\end{aligned}
$$

Similarly, $w_{n}(\sigma)$ of (5.12) is given by

$$
w_{n}(\sigma)=\frac{1}{\sqrt{2 n}} \operatorname{Im} \oint_{z=0} \frac{d z}{2 \pi i} \frac{h^{\prime}(z)}{z^{n}}\left\{L_{1}(h(z))+L_{2}(h(z))-L_{1}\left(h\left(e^{i \sigma}\right)+h(z)\right)+L_{2}\left(h\left(e^{i \sigma}\right)-h(z)\right)\right\} .
$$

The functions $L_{1}(h)$ and $L_{2}(h)$ are expressed in terms of the gamma functions as

$$
L_{1}(h)=-\frac{2 \sin h}{\pi^{3 / 2}}\left\{\Gamma\left(1+\frac{h}{\pi}\right) \Gamma\left(\frac{1}{2}-\frac{h}{\pi}\right)+\Gamma\left(1-\frac{h}{\pi}\right) \Gamma\left(\frac{1}{2}+\frac{h}{\pi}\right)\right\},
$$

and

$$
L_{2}(h)=-\frac{2 \cos h}{\pi^{3 / 2}}\left\{\Gamma\left(1+\frac{h}{\pi}\right) \Gamma\left(\frac{1}{2}-\frac{h}{\pi}\right)-\Gamma\left(1-\frac{h}{\pi}\right) \Gamma\left(\frac{1}{2}+\frac{h}{\pi}\right)\right\} .
$$

The expansions (5.11) and (5.13) around the midpoint are obtained by using the definition (2.22) of the parameter $s$ and the Stirling formula for the gamma function.

\section{References}

[1] A. Sen, "Descent relations among bosonic D-branes," Int. J. Mod. Phys. A 14, 4061 (1999) arXiv:hep-th/9902105. 
[2] A. Sen and B. Zwiebach, "Tachyon condensation in string field theory," JHEP 0003, 002 (2000) arXiv:hep-th/9912249.

[3] E. Witten, "Noncommutative Geometry And String Field Theory," Nucl. Phys. B 268, 253 (1986).

[4] J. A. Harvey and P. Kraus, "D-branes as unstable lumps in bosonic open string field theory," JHEP 0004, 012 (2000) arXiv:hep-th/0002117.

[5] R. de Mello Koch, A. Jevicki, M. Mihailescu and R. Tatar, "Lumps and p-branes in open string field theory," Phys. Lett. B 482, 249 (2000) arXiv:hep-th/0003031.

[6] N. Moeller, A. Sen and B. Zwiebach, "D-branes as tachyon lumps in string field theory," JHEP 0008, 039 (2000) arXiv:hep-th/0005036.

[7] L. Rastelli, A. Sen and B. Zwiebach, "String field theory around the tachyon vacuum," Adv. Theor. Math. Phys. 5, 353 (2002) arXiv:hep-th/0012251.

[8] L. Rastelli, A. Sen and B. Zwiebach, "Classical solutions in string field theory around the tachyon vacuum," Adv. Theor. Math. Phys. 5, 393 (2002) arXiv:hep-th/0102112.

[9] L. Rastelli, A. Sen and B. Zwiebach, "Boundary CFT construction of D-branes in vacuum string field theory," JHEP 0111, 045 (2001) arXiv:hep-th/0105168.

[10] P. Mukhopadhyay, "Oscillator representation of the BCFT construction of D-branes in vacuum string field theory," JHEP 0112, 025 (2001) arXiv:hep-th/0110136.

[11] G. W. Moore and W. Taylor, "The singular geometry of the sliver," JHEP 0201, 004 (2002) arXiv:hep-th/0111069.

[12] H. Hata and S. Moriyama, "Observables as twist anomaly in vacuum string field theory," JHEP 0201, 042 (2002) arXiv:hep-th/0111034.

[13] H. Hata, S. Moriyama and S. Teraguchi, "Exact results on twist anomaly," JHEP 0202, 036 (2002) arXiv:hep-th/0201177.

[14] H. Hata and T. Kawano, "Open string states around a classical solution in vacuum string field theory," JHEP 0111, 038 (2001) arXiv:hep-th/0108150.

[15] L. Rastelli, A. Sen and B. Zwiebach, "A note on a proposal for the tachyon state in vacuum string field theory," JHEP 0202, 034 (2002) arXiv:hep-th/0111153.

[16] H. Hata and H. Kogetsu, "Higher level open string states from vacuum string field theory," JHEP 0209, 027 (2002) arXiv:hep-th/0208067. 
[17] L. Rastelli, A. Sen and B. Zwiebach, "Half strings, projectors, and multiple D-branes in vacuum string field theory," JHEP 0111, 035 (2001) arXiv:hep-th/0105058.

[18] D. J. Gross and W. Taylor, "Split string field theory. I," JHEP 0108, 009 (2001) arXiv:hep-th/0105059.

[19] D. J. Gross and W. Taylor, "Split string field theory. II," JHEP 0108, 010 (2001) arXiv:hep-th/0106036.

[20] K. Furuuchi and K. Okuyama, "Comma vertex and string field algebra," JHEP 0109, 035 (2001) arXiv:hep-th/0107101.

[21] V. A. Kostelecky and R. Potting, "Analytical construction of a nonperturbative vacuum for the open bosonic string," Phys. Rev. D 63, 046007 (2001) arXiv:hep-th/0008252.

[22] E. Fuchs, M. Kroyter and A. Marcus, "Continuous half-string representation of string field theory," JHEP 0311, 039 (2003) arXiv:hep-th/0307148.

[23] L. Rastelli, A. Sen and B. Zwiebach, "Star algebra spectroscopy," JHEP 0203, 029 (2002) arXiv:hep-th/0111281.

[24] B. Feng, Y. H. He and N. Moeller, "The spectrum of the Neumann matrix with zero modes," JHEP 0204, 038 (2002) arXiv:hep-th/0202176.

[25] D. Gaiotto, L. Rastelli, A. Sen and B. Zwiebach, "Ghost structure and closed strings in vacuum string field theory," Adv. Theor. Math. Phys. 6, 403 (2003) arXiv:hep-th/0111129.

[26] D. Gaiotto, L. Rastelli, A. Sen and B. Zwiebach, "Star algebra projectors," JHEP 0204, 060 (2002) arXiv:hep-th/0202151.

[27] K. Okuyama, "Ratio of tensions from vacuum string field theory," JHEP 0203, 050 (2002) arXiv:hep-th/0201136.

[28] K. Okuyama, "Ghost kinetic operator of vacuum string field theory," JHEP 0201, 027 (2002) arXiv:hep-th/0201015. 